\title{
Solar Absolute Reference Spectrum
}

\author{
By HEINZ NECKEL
}

Hamburg Observatory, Gojenbergsweg 112, D-21029 Hamburg, Germany

Requirements and problems in establishing an absolute solar spectrum, which could serve equally well all concerned sciences (e.g. solar physics, atmospheric physics, astrophysics) as a qualified reference, are illustrated by means of a high resolution spectral atlas with absolute intensity and irradiance scales. Also the organizational aspects are pointed out: the desire for one worldwide reference spectrum involves its recommendation by a competent team of scientists and the agreement of the relevant, official organizations.

\section{Introduction}

In the first half of this century inquiries for an absolute solar reference spectrum could be easily answered: the work of Abbot and his co-workers had provided spectral irradiance data of unique and undoubted quality. Even without official recommendation, these data were adopted for most hand and text books and were used by scientists and engineers everywhere on the world.

Since then, many new absolute measurements of solar radiation were made. They often differed, however, in important aspects (e.g. in bandwidth) and yielded - at least partly very inconsistent results. To be able to relate all relevant data to a common base, and also to satisfy all those who are interested in absolute radiation data, the recommendation of a generally accepted reference spectrum appears to be highly desirable. I think it is this desire which let the organizers of this colloquium propose the topic of this contribution.

About 20 years ago there was already one attempt to establish something like a 'solar absolute reference spectrum'. The procedure to establish this reference was exemplary, and it is worthwhile to quote the relevant passages from Thekaekara (1975): 'An ad hoc committee ... was ... appointed ... to study this question. The Committee made a detailed survey of all available information and recommended standard values for the solar constant and solar spectrum. ... These values have been formally adopted by the American Society for Testing and Materials (ASTM) as the "Standard Specification for Solar Constant and Air Mass Zero Solar Spectral Irradiance". ...'. Actually, this 'NASA/ASTM Standard of Total and Spectral Solar Irradiance' was primarily based on the results from the GALILEO experiment aboard the NASA Convair 990 aircraft. Curiously, it ranked among the least reliable irradiance data which have been published 'after Abbot', and therefore never played the role of a generally accepted reference. Nevertheless, the quoted 'recipe' how to establish a reference spectrum is still valid, but with associations like IAU (International Astronomical Union) and IAMAP (International Association of Meteorology and Atmospheric Physics) as the responsible organizations.

The more important problem is, of course, the realization of an absolute spectrum which is reliable enough for recommendation and adoption as a worldwide reference. I will demonstrate the requirements - and the involved problems - by means of a special, unpublished spectral atlas, which gives the solar intensities in absolute values. The main characteristic of this atlas is that it actually deals with two spectra, one of the diskcenter and one of the disk-averaged radiation. Clearly, also the desire for a reference spectrum concerns not only the flux, but the intensity as well. Therefore, any official agreement should refer to a comparable set of such two consistent spectra. 

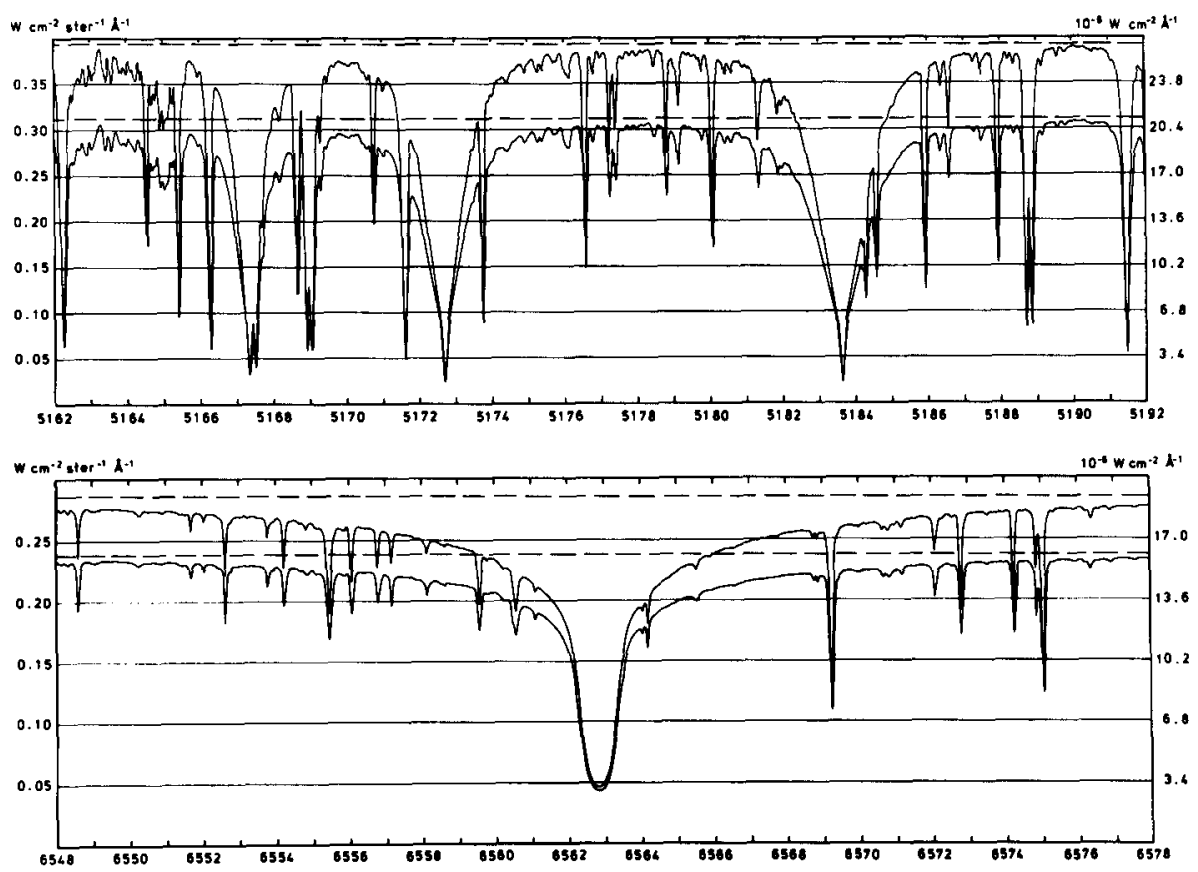

Figure 1. Two extracts from an absolutely scaled solar spectrum atlas for disk-center (upper curve) and disk-averaged (lower curve) intensity (left scale) Right scale: irradiance at $1 \mathrm{AU}$ (lower curve). Dashed lines indicate the levels of the corresponding (quasi) continua.

First, I will describe the final product and point to its specialities, and then make a few comments on the problems with respect to the mixing of its 'ingredients'.

\section{Absolute spectra of disk center and disk-averaged radiation}

Figure 1 displays two extracts from the atlas, which actually extends from 329 to $1251 \mathrm{~nm}$ and is stored on magnetic tape. The left scales are in $\mathrm{W} \mathrm{cm}^{-2} \mathrm{sr}^{-1} \AA^{-1}$ and provide for both, disk center and disk-averaged spectra, the absolute intensities. The right scales are in $\mu \mathrm{W} \mathrm{cm} \mathrm{cm}^{-2} \AA^{-1}$ and refer only to the lower spectra on each panel, which represent the spectral irradiance at $1 \mathrm{AU}$. The atlas includes also the quasi-continuum levels (dashed lines), which are defined by the highest intensities in the two overall spectra (Figure 2).

The spectra are based on Fourier Transform Spectra (FTS), which were obtained by Brault and his co-workers at the Kitt Peak National Solar Observatory (Brault 1979; the disk spectra are copies from the same original data which were used by Kurucz et al. 1984 to prepare their Solar Flux Atlas). The main characteristics of these spectra are:

(1) The spectral resolution, which depends solely on the maximum distance of travel of the retro-reflectors, was for all spectra about $350000(10 \mathrm{~m} \AA$ at $350 \mathrm{~nm})$.

(2) The dispersion (= distance between two successive recorded spectral data) changes from $4 \mathrm{~m} \AA$ at $330 \mathrm{~nm}$ to $20 \mathrm{~m} \AA$ at $1250 \mathrm{~nm}$.

(3) Scattered light is unimportant, since it is unmodulated and not detected as signal.

(4) If the bandwidth is limited to the upper third of the available range, the intensity scale is perfectly linear even with a nonlinear detector.

(5) There may be, however, minor errors in the zero points of the intensity scale, which 


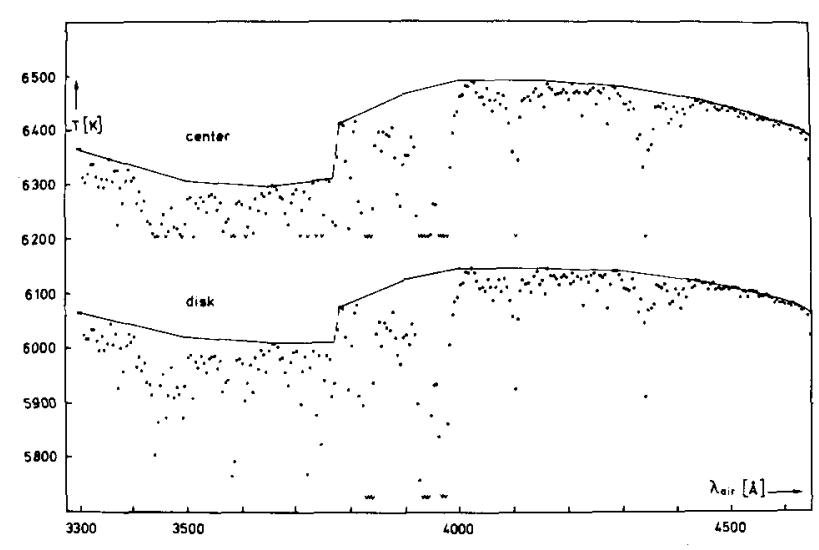

FIGURE 2. Radiation temperatures from intensity maxima in $5 . \AA$ wide spectral intervals of disk center and disk-veraged spectra. Polygonal tracks indicate the levels of the adopted 'quasi-continuum'.

can be caused by errors of the height of the central peaks in the interferograms due to noise.

(6) After each solar spectrum the (continuous) spectrum of a reference lamp was recorded and any further treatment, e.g. the absolute calibration of the intensity scales, referred to the ratio spectra Sun/lamp.

The intensity scale was calibrated by means of the absolute data of Neckel \& Labs (1984) for spectral bands being precisely $20.0 \AA$ wide. It should be noted that the data for both disk-center and disk-averaged intensities are based on the same observations of the disk center. These were later supplemented by special observations of the centerto-limb variation of the $20 \AA$ bands by Neckel \& Labs (1984), which provided then the correct ratios of disk-averaged to disk-center intensities F/I (Neckel \& Labs 1984 for $\lambda<660 \mathrm{~nm}$ and Pierce \& Slaughter 1977 or Pierce et al. 1977 for $\lambda>660 \mathrm{~nm}$ ).

Figure 3 displays the resulting calibration curves and the residuals resulting from the least square fits. The overall spectral distribution of the curves reflects basically the spectrum of the reference lamp. The scatter of the residuals is usually about $0.5 \%(1 \sigma)$.

The wavelength-scale refers to wavelengths in air, is corrected for radial velocity and instrumental alignment errors and is in the system of the Kitt Peak Tables of Pierce \& Breckinridge (1973). The remaining wavelength errors range from $0.2 \mathrm{~m} \AA$ at $330 \mathrm{~nm}$ to $2 \mathrm{~m} \AA$ at $1250 \mathrm{~nm}$. So, the atlas spectra can be used as reference for intensities as well as for wavelength differences and line profiles.

\section{Consistency with other solar and stellar data}

Within the limits set by intrinsic variations of the intensity distribution across the solar disk (see below), the atlas ratios of disk-averaged to disk-center intensity agree at continuum wavelengths with those which follow from the limb darkening data of Pierce \& Slaughter (1977) for $\lambda<730 \mathrm{~nm}$, of Pierce et al. (1977) for $\lambda>740 \mathrm{~nm}$, and of Neckel \& Labs (1994) for $330<\lambda<1099 \mathrm{~nm}$. The complete integral of the irradiance spectrum (330 to $1250 \mathrm{~nm}$ ) is $1.06 \mathrm{~kW} \mathrm{~m}^{-2}$ and corresponds to a solar constant of about $1.37 \mathrm{~kW} \mathrm{~m}^{-2}$. The irradiance spectrum also agrees almost perfectly with the most recent model prediction of Kurucz (1992) where the opacities resulted from atomic and 


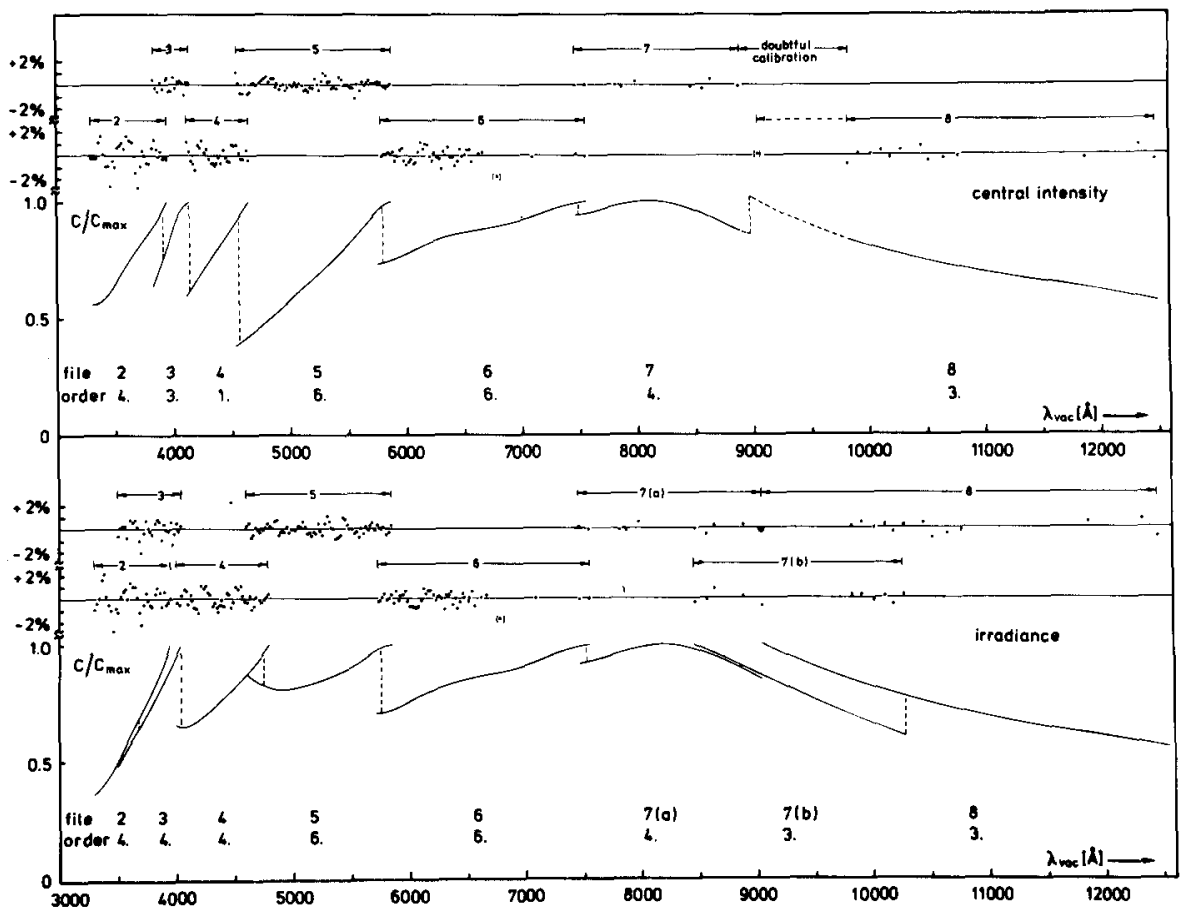

Figure 3. Calibration curves for Kitt Peak FTS spectra (ratio Sun to reference lamp), in units of their maximum values. Polynomials of order 1 to 6 were fitted to the ratios between observed absolute intensity integrals being exactly $20 \AA$ wide and the corresponding FTS integrals. The residuals of the least square fits are plotted above the curves. Their standard deviations range between 0.3 and $1.1 \%$, the average is $0.5 \%$.

molecular line data of some 58 million lines with a mixing-length to scale-height ratio of 1.25 , and micro-turbulent velocity of $1.5 \mathrm{~km} / \mathrm{s}$. This agreement is obvious from Figure 3 which was adopted from Kurucz (1992).

A comparison of the irradiance spectrum with the absolute flux of a solar type star (16 Cyg B) is given in Figure 5. The upper plot displays the residuals with respect to the overall, $1 / \lambda$-proportional gradient shown in plot 2 , which indicates that $16 \mathrm{Cyg} \mathrm{B}$ is slightly cooler than the Sun $(\approx 40 \mathrm{~K})$. Besides this gradient are no other significant systematic differences. Only at a few wavelengths one or the other (but never both) stellar value deviates by more than 0.02 magnitudes - or $2 \%$ - from the solar data. It should be noted that this Sun-star comparison avoids any passband mismatches, since the solar data refer precisely to the same passband that were used by the stellar observers. Even the errors in the wavelength settings of the stellar observations could be evaluated precisely. Assuming errors of $1 \AA$ for the Hardorp (1980) data and between 0 and $4 \AA$ for the Taylor (1984) data reduced not only the scatter in the Sun-star differences significantly, but improved also very clearly the agreement between both stellar data sets. This is a good example for the advantage of using a high resolution spectrum as reference.

The Sun-star comparison provided also rather precise data for the Sun's colors (U-B, B-V), which agree well with those of other solar type stars and correspond precisely to the Sun's spectral type as shown in Table 1 . In the spectral band which defines the visual magnitudes ' $\mathrm{V}$ ', the ratio solar irradiance to flux of $16 \mathrm{Cyg} \mathrm{B}$ is $1.52 \times 10^{13}$ (magnitude 


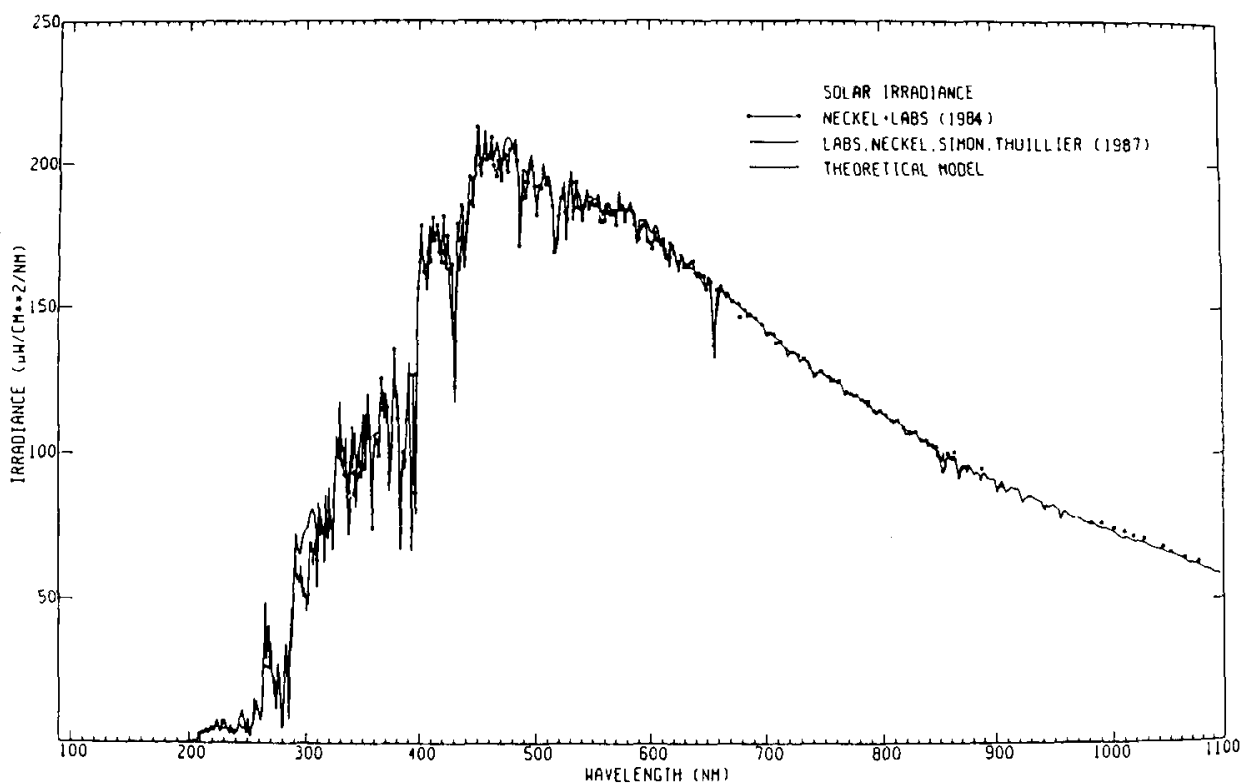

FIgURE 4. Predicted solar irradiance from Kurucz (1992) compared to the observed data of Labs et al. (1987) for $\lambda<360 \mathrm{~nm}$ and Neckel \& Labs (1984) for $\lambda>330 \mathrm{~nm}$. The latter data provided the absolute scale for the atlas irradiance spectrum.

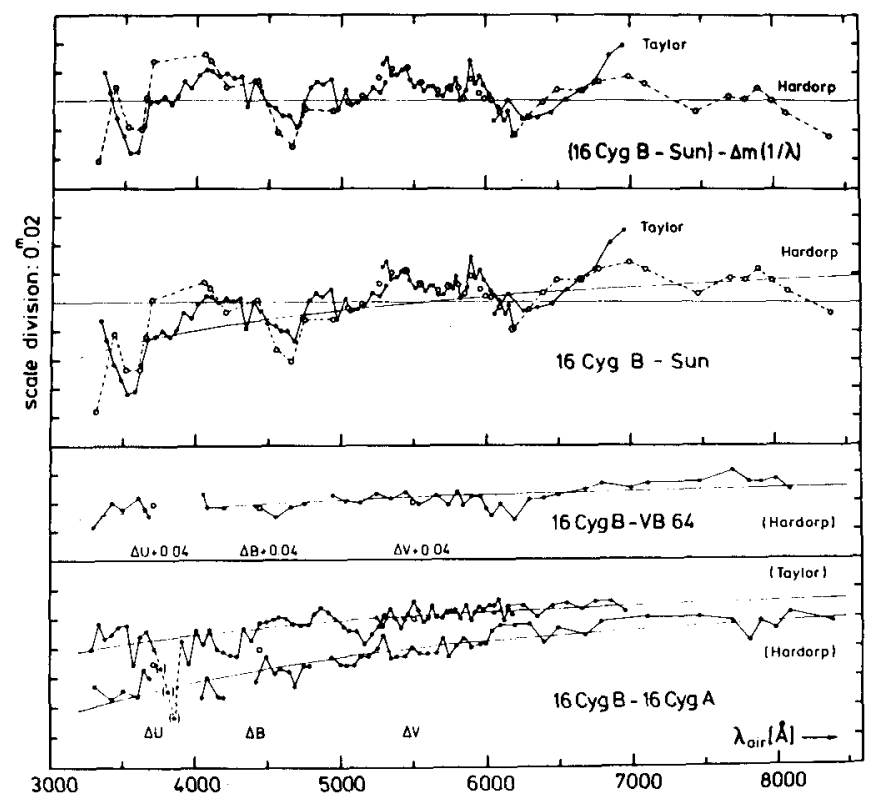

Figure 5. Comparison of the flux of the solar type star 16 Cyg B with the solar irradiance spectrum and with the fluxes of two other 'solar analogs'. The stellar data are from Hardorp (1980) and Taylor (1984) and are in the system defined by the absolute flux data of the standard star Vega provided by Hayes (1985). For details see Neckel (1986). 


$\begin{array}{ccccc}\text { Star } & \mathrm{T}_{\text {eff }} & \mathrm{U}-\mathrm{B} & \mathrm{B}-\mathrm{V} & \text { Spectral type } \\ \text { 16 Cyg B } & 5736 & 0.204 & 0.658 & \text { G3 V } \\ \text { VB 64 } & 5758 & 0.200 & 0.654 & \text { G2+ V } \\ \text { Sun } & 5777 & 0.195 & 0.650 & \text { G2 V } \\ \text { 16 Cyg A } & 5804 & 0.190 & 0.644 & \text { G1.5 Vb }\end{array}$

TABLE 1. Consistent spectral characteristics of Sun and solar type stars. Stellar colors are from Blanco et al. (1966) or Nicolet (1978); MK spectral types from Keenan \& Yorka (1985) (N.B.: all three stars and the Sun are MK standards).

difference $\Delta \mathrm{V}=32.95$ ). With $\mathrm{V}_{16 \mathrm{Cyg}}=6.20$ (and $\mathrm{V}_{\text {Vega }}=0.03$ ) one gets for the Sun's apparent visual magnitude $\mathrm{V}_{\mathrm{Sun}}=-26.75$, one of the familiar text book values.

\section{Problems and restrictions}

The main restriction for preparing a high resolution absolute reference spectrum comes, of course, from the Earth's atmosphere, which not only fixes the lower wavelength limit (here: $329 \mathrm{~nm}$; actually, the atlas range could be extended down to about $300 \mathrm{~nm}$ ), but also blends the red and infrared spectral region with terrestrial lines and absorption bands or even blocks completely wide spectral regions. As far as ground-based observations are concerned, the precise determination of atmospheric extinction is another, special problem. While absolute broad band measurements of the solar irradiance from space seem to be already a matter of standard routine, high resolution spectra of FTS quality and absolute intensity measurements will for some time remain a matter of ground based observations. Another problem arises from the Sun's variability, which prevents the provision of homogeneous data and limits so the accuracy of any final spectral reference. With respect to the wavelength scale it should be noted that in high resolution spectra like the Kitt Peak FTS not only line profiles and central line depths, but also even the wavelengths vary on time scales of the order of hours and days (disk center: $500 \mathrm{~m} / \mathrm{s}$, full disk: $50 \mathrm{~m} / \mathrm{s}$; see e.g. Neckel \& Labs 1990). Time scales between minutes and weeks are found also for variations of the intensity distribution across the disk. In particular, for wide spectral bands including strong absorption lines the ratios disk-averaged to diskcenter intensity $(\mathrm{F} / \mathrm{I})$ may undergo variations in the order of $1-2 \%$ (occasionally even more!) of the disk center intensity (Neckel \& Labs 1987, 1994). Clearly, such variations of $\mathrm{F} / \mathrm{I}$ indicate mainly local intensity variations, e. g. at disk center, and do not necessarily point to variations of the overall flux. Therefore, absolute fluxes may be a better base for the construction of absolute reference spectra than absolute intensities. In any case, ideal reference spectra would have to be derived from data which either all refer to the same instant of time, or are all representative averages for the same, sufficiently large time interval, possibly near activity minimum. A look at Table 2 reveals that the absolutely calibrated Kitt Peak FTS spectra are far from such ideal combination: the observing days of the contributing data are distributed randomly over a period of about 20 years.

After all, one has to realize that a homogeneous, absolute solar spectrum of high spectral resolution, which includes the major spectral region of interest $(200 \mathrm{~nm}-10 \mu \mathrm{m}$ corresponding to $99.9 \%$ of all radiation) is not yet available and can not be constructed from available observational data. Therefore, any agreement on a reference spectrum must include one or another compromise, which has to be based on different data coming from different observations (ground based and space borne) made at different times. But such an agreement should not only refer to the spectral reference, but to all sorts of solar 


\begin{tabular}{ccrccc} 
& \multicolumn{2}{c}{ FTS(center) } & \multicolumn{2}{c}{ FTS(disk) } & \\
& $\lambda(\mathrm{nm})$ & dates & $\lambda(\mathrm{nm})$ & dates & \\
& $329-391$ & $1979 / 12 / 28$ & $329-368$ & $1981 / 6 / 21$ & \\
& $391-414$ & $1980 / 2 / 15$ & $368-404$ & $/ 6 / 22$ & \\
& $414-456$ & $/ 2 / 15$ & $404-475$ & $1980 / 11 / 23$ & \\
& $456-580$ & $/ 3 / 30$ & $475-576$ & $1981 / 3 / 24$ & \\
& $580-749$ & $1981 / 5 / 12$ & $576-753$ & $/ 3 / 25$ & \\
& $749-897$ & $/ 5 / 13$ & $753-1028$ & $/ 3 / 25$ & \\
& $897-1251$ & $/ 5 / 13$ & $1028-1251$ & $/ 5 / 11$ & \\
$\lambda(\mathrm{nm})$ & I(center) & & & F $/$ I & \\
$329-413$ & $1964 / 4 / 26-5 / 13$ & 4 & $319-660$ & $1981 / 4 / 14-30$ & NL \\
$401-657$ & $1961 / 9 / 1-10 / 14$ & 8 & $660-730$ & $1974 / 3-1975 / 1$ & PS \\
$639-1248$ & $1962 / 9 / 9-10 / 2$ & 10 & $730-1251$ & $1974 / 9-1976 / 3$ & PSW
\end{tabular}

TABLE 2. Observation days for atlas data collection. The references NL, PS and PSW refer to Neckel \& Labs (1984), Pierce \& Slaughter (1977) and Pierce et al. (1977), respectively.

and stellar radiation data, including solar total irradiance, stellar colors, limb darkening, model predictions, etc. One basic requirement for the spectral reference is its internal consistency, which can be easily tested by means of the Kitt Peak FTS or any other comparable high resolution spectra (see Figure 3).

\section{REFERENCES}

Blanco, V. M., Demers, S., Douglass, G, G. \& FitzGerald, M.P. 1966 Photoelectric catalog. Publ. U. S. Naval Obs., pp. 21.

Brault, J. W. 1979 Solar Fourier Transform spectroscopy. In Proceedings of JOSO Workshop pp. 33. Osservazioni e memorie dell'Osservatorio Astrofisico di Arcetri 106.

Hardorp, J. 1980 The Sun amoung the stars III: Energy distribution of 16 G-type stars and the solar flux calibration Astron. Astrophys. 91, 221.

HAYES, D. S. 1985 Stellar absolute fluxes and energy distributions from 0.32 to $4.0 \mu \mathrm{m}$. In Calibration of Fundamental Stellar Quantities, IAU Symp. No 111 (ed. D.S. Hayes, L.E. Pasinetti \& A.D. Philip). pp. 225. D. Reidel Publ. Co..

Keenan, P. C. \& Yorka, A. R. 19851986 revised MK spectral standards: Stars G0 and later. Standard Star Newsletter 6, 2.

Kunucz, R. L. 1992 Model atmospheres for population synthesis. In The Stellar Populations of Galaxies, IAU Symp. No 149 (ed. B. Barbuy \& A. Renzini). pp. 225. Kluwer Academic Publishers.

Kurucz, R. L., Furenlid, I., Brault, J. \& Testermann, L. 1984 Solar Flux Atlas from 296 to $1300 \mathrm{~nm}$ Harvard University Press.

Labs, D., Neckel, H., Simon, P. C. \& Thuillier, G. 1987 Ultraviolet solar irradiance measurements from 200 to $358 \mathrm{~nm}$ during Spacelab 1 mission. Solar Phys. 107, 203.

NECKEL, H. 1986 The absolute energy distributions of the Sun, of the solar analogs 16 Cyg B, Hyades VB 64, 16 Cyg A, and of the standard stars $\alpha$ Lyr and 29 Psc. Astron. Astrophys. $159,175$.

NECKEL, H. \& LABS, D. 1984 The solar radiation between 3300 and $12500 \AA$. Solar Phys. 90, 205.

Neckel, H. \& LABs, D. 1987 Asymmetry and variations of solar limb darkening along the diameter defined by diurnal motion in April 1981 (and later). Solar Phys. 110, 139.

NeCKEL, H. \& LABs, D. 1990 Variations of 'wavelengths' and 'bisector indices' of 70 solar 
spectral lines between 3300 and $3960 \AA$ in Kitt Peak FTS spectra. Solar Phys. 126, 207. NeCKel, H. \& LaBs, D. 1994 Solar limb darkening 1986-1990 ( $\lambda \lambda 303$ to $1099 \mathrm{~nm}$ ). Solar Phys., submitted.

NICOLET, B. 1978 Catalog of homogeneous data in the UBV photoelectric photometric system. Astron. Astrophys. Suppl. 34, 1.

Pierce, A. K. \& Breckinridge, J. B. 1973 The Kitt Peak Table of Photographic Solar Spectrum Wavelengths Kitt Peak Nat. Obs. Contribution No. 559.

Pierce, A. K. \& Slaughter, C. D. 1977 Solar limb darkening I: $\lambda \lambda 3033-7297 \mathrm{~nm}$. Solar Phys. $51,25$.

Pierce, A. K., Slaughter, C. D. \& Weinberger, D. 1977 Solar limb darkening in the interval 7404-24018 ̊. Solar Phys. 52, 179.

TAYLOR, B. J. 1984 Energy distribution for 1 solar-type stars and synthetic UBV photometry of the Sun. Astrophys. J. Suppl. 54, 167.

Thekaekara, M. P. 1975 The total and spectral solar irradiance and its possible variations. In The Solar Constant and the Earth's Atmosphere (ed. H. Zirin \& J. Walter). pp. 232. Boulder, CO., USA. 